\title{
Stress Relaxation and Evolution of Surface Microstructure During Tensile and Compressive Loading of Polymer Nanocomposites
}

\author{
S. Shanmugam $*^{1,2}$ and R. D. K. Misra*1,2 \\ ${ }^{* 1}$ Center for Structural and Functional Materials \\ ${ }^{* 2}$ Department of Chemical Engineering \\ University of Louisiana at Lafayette, Lafayette, LA 70504-4130, USA
}

The high susceptibility of polymeric materials to surface deformation during tensile and compressive loading conditions is a disadvantage in applications requiring optical clarity because they exhibit a 'stress whitening.' The common inherent factors that influence stress whitening or opacity include length of molecular chains, crystallinity, and isotacticity [1]. In this regard, polymer nanocomposites are radical alternatives to the conventional filled polymers or polymer blends from the viewpoint of mechanical properties and ability to retain optical transparency.

The paper describes a comparative study of the mechanical response of neat and clay-reinforced polymer nanocomposites during tensile and compressive loading using scanning electron microscopy (SEM). During tensile loading, neat polymers exhibit localized stress relaxation centers that are characterized by symmetrical psiloma-type of surface deformation features at low strains. At higher strains, ridges/wrinkles are nucleated within the stress relaxed zones that encourage plastic flow and tearing leading to fibrillation (Figure 1). These severely deformed fibrils increase opacity. The reinforcement with nanoclay reduces the size and density of stress relaxation zones with consequent reduction of plastic flow.

During compressive loading, the micromechanism of surface deformation is retained in neat polymers and clay-reinforced polymer nanocomposites (Figure 2). However, the intensity of surface deformation is significantly reduced with 'ironing' being the dominant mode of deformation in polymer nanocomposites. The optical transparency is retained because the build-up of the stress in the matrix is minimized, eliminating deformation features responsible for lower light scattering efficiency. The reduced intensity of surface deformation in polymer nanocomposites is ascribed to the reinforcement effect of clay particles that increases the modulus of the composite rather than the clay-induced structural changes such as breaking of the interconnected spherulitic structure.

Another interesting aspect is that the evolution of microstructure during tensile and compressive loading is retained in polymer nanocomposites irrespective of the structural changes induced in the polymer matrix by clay particles. These observations suggest that even during the compressive loading experienced during scratching, the tensile component of the applied stress has a strong influence on the micromechanism of deformation.

Reference:

1. A. Dasari and R.D.K. Misra, Acta Materialia 52 (2004) 1683. 


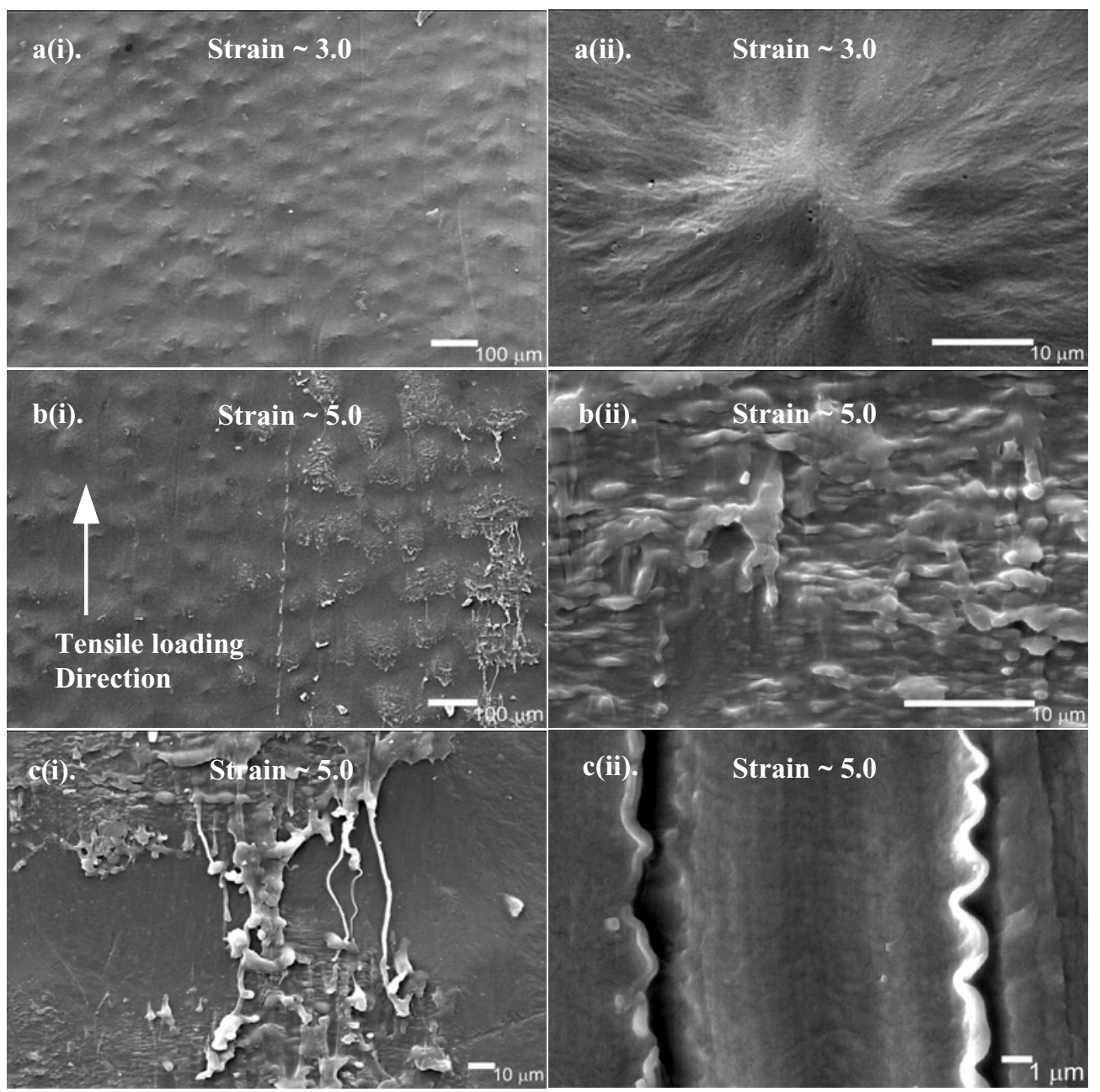

Figure 1: SEM micrographs of neat polybutene showing psiloma-type stress relaxation centers (a) containing ridges (b) that encourage tearing leading to fibrillation (c). The tensile test was carried out at displacement rate of $\sim 50 \mathrm{~mm} / \mathrm{min}$ ( strain rate of $\sim 0.016 \mathrm{~s}^{-1}$ ).

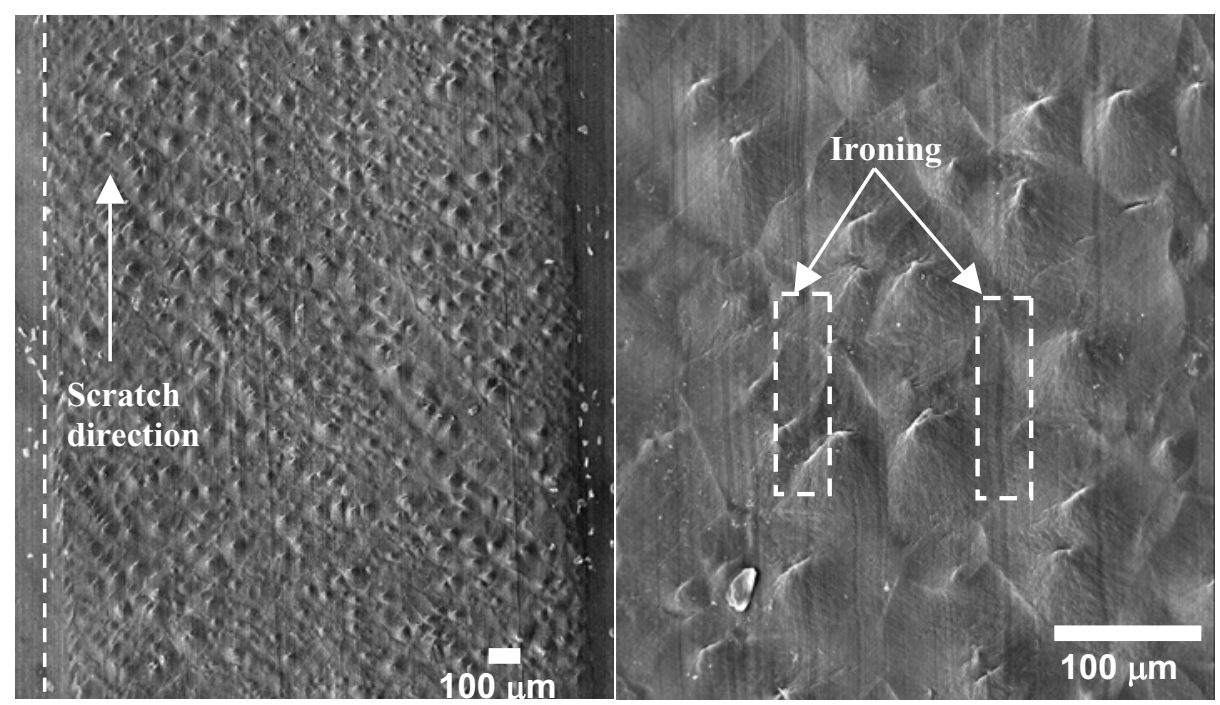

Figure 2: SEM micrographs of psiloma and ironing mode of deformation in neat PB during compressive loading. The test load was $4 \mathrm{~kg}$. 